\title{
Long-term results measured by BREAST-Q reveal higher patient satisfaction after "autoimplant-mastopexy" than augmentation-mastopexy
}

\author{
Lisanne Grünherz, Anna Burger, Pietro Giovanoli, Nicole Lindenblatt \\ Department of Plastic and Reconstructive Surgery, University Hospital Zurich, Zurich, Switzerland \\ Contributions: (I) Conception and design: All authors; (II) Administrative support: All authors; (III) Provision of study materials or patients: All \\ authors; (IV) Collection and assembly of data: All authors; (V) Data analysis and interpretation: All authors; (VI) Manuscript writing: All authors; (VII) \\ Final approval of manuscript: All authors. \\ Correspondence to: Nicole Lindenblatt, MD. Department of Plastic and Reconstructive Surgery, University Hospital Zurich, Raemistrasse 100,8091 \\ Zurich, Switzerland. Email: nicole.lindenblatt@usz.ch.
}

\begin{abstract}
Background: The aim of a mastopexy is to raise the breast projection, tighten the skin envelope, and place the nipples in an optimal position in projection to the inframammary fold. This procedure is often combined with prosthetic implants or an autoimplant. Given the current increasing demand for the use of autologous tissue, we evaluated mastopexies combined with either an autoimplant or prosthetic implant in terms of patient satisfaction and long-term results.

Methods: We evaluated 34 patients who underwent a mastopexy with simultaneous breast prosthesis or an autoimplant. During follow-ups we obtained standardized breast measurements, BREAST-Q score, and preand postoperative photographs to perform photometric measurements.

Results: BREAST-Q score of patients that underwent autoimplant-mastopexies revealed a higher patient satisfaction with significant differences in satisfaction with breast appearance $(69 \pm 18 v s .55 \pm 16, \mathrm{P}=0.03)$ and outcome ( $71 \pm 18$ vs. $48 \pm 26, \mathrm{P}=0.009)$. Regarding breast shape, photometric evaluations presented a significantly different breast shape with higher upper pole fullness in augmentation-mastopexy patients. No statistical significance between long-term results and complication rates could be observed in either patient groups.

Conclusions: The BREAST-Q score implies a higher overall long-term satisfaction in patients that received autoimplant-mastopexy with similar long-term results compared with augmentation-mastopexy. Nevertheless, individual decision-making is necessary and should be based on the degree of ptosis, existing breast volume, previous operations, and patients' preferences regarding postoperative breast shape and projection.
\end{abstract}

Keywords: Breast ptosis; mammaplasty; patient satisfaction

Submitted May 07, 2019. Accepted for publication Aug 28, 2019.

doi: $10.21037 /$ gs.2019.09.05

View this article at: http://dx.doi.org/10.21037/gs.2019.09.05

\section{Introduction}

Breast ptosis is a common issue in plastic surgery that may result from a combination of breast parenchyma involution and reduced elasticity of the skin envelope. This therefore leads to a low breast profile and nipple-areolar complex (NAC) that descends towards the inframammary crease.
Besides aging, several factors can contribute to breast ptosis such as massive weight loss, pregnancies, breast-feeding, and postpartum involution (1).

A variety of mastopexy techniques have been described to address the degrees of breast ptosis (2-5). These approaches aim to raise the breast projection, tighten the skin envelope, 
and place the NAC in an optimal position perpendicular to the inframammary crease. However, in patients with extensive breast tissue loss, a mastopexy often needs to be combined with prosthetic implants in order to restore the projection of the breast and gain a sufficient upper pole fullness. While breast implants cause a foreign body reaction that can lead to capsular contracture and results in poor aesthetic results, and pain in the long run, several authors have proposed the use of glandular rearrangement to optimize central mound projection in effort to avoid the use of implants (6-8).

In the past, various techniques that differ in the incision pattern and dermoglandular pedicle have been proposed under the term "Autoaugmentation" mastopexy $(3,4,8,9)$. In our department, an inferior based dermoglandular flap proposed by Graf and Biggs was used (2). Although all techniques make use of the existing breast tissue, we prefer to use the term "Autoimplant-Mastopexy," as no augmentation of the breast volume was achieved. Concerning mastopexies combined with an autoimplant, literature is relatively sparse and heterogeneous due to different surgical techniques and outcome definitions (1-4). Given the increasing demand of the use of autologous tissue, we therefore aimed to compare the combination of a mastopexy either with an "Autoimplant" or a silicone implant to determine differences in complications, longterm results and patient satisfaction using BREAST-Q score (10). To demonstrate our comparison between both techniques, we used the term "Mastopexy" to refer to an "Autoimplant-Mastopexy."

\section{Methods}

\section{Patient evaluation}

This study was approved by the Cantonal Ethics Committee of Zurich, Switzerland (Ethical approval No. 2017-01333). Surgeries were performed at the Department of Plastic and Reconstructive Surgery at the University Hospital Zurich. We included 34 patients that were treated in our division from $04 / 2011$ and $05 / 2017$. The inclusion criteria were defined as follows: patients $\geq 18$ years of age who underwent a mastopexy with simultaneous breast prosthesis augmentation or autoimplant that took place in our clinic. All patients gave written consent prior to study participation.

After a minimum of 1 year, a follow-up consultation included standardized breast measurements, standardized postoperative photographs, and BREAST-Q scoring took place. Standardized breast measurements included sternal notch to nipple distance (SNN), nipple to inframammary fold distance, midline to nipple distance, diameter of nipple areolar complex, and breast width. The study protocol provides an examination of all patients by the first author. Patients' demographics were registered and evaluated according age, body mass index (BMI), smoking habits and their past medical history.

Additional photometric measurements with Mirror 7.1.1 imaging software (Canfield Scientific, Fairfield, NJ, USA) were performed on pre- and postoperative photographs in a lateral view with the same focal distance and body position in 18 patients.

\section{Surgical technique}

In all cases, the technique described by Graf and Biggs was adapted to create an autoimplant (2). Patients were marked in a standing position prior to surgery as per standard protocol. The operation proceeded with the deepithelialization of the Wise pattern (Figure 1A), followed by horizontal incision of the dermis about one centimeter below the areola, perpendicular to the plane of the thoracic wall until the incision meets it at the fourth intercostal space (Figure $1 B$ ). In the upper portion of the flap, an oblique incision was made, therefore leaving intact tissue for the breast pillars laterally and medially. The lower portion of the flap was dissected down to the original inframammary crease widening at its base, thus creating an inferior pedicle flap (Figure 1C). Blood supply was based on the arteries of the fourth and fifth intercostal spaces. After a bipedicled pectoralis major sling was prepared, the flap was placed under the muscle and fixed to the thoracic wall, followed by closure of the breast pillars, as well as suturing of the dermis and skin plane-by-plane, which resulted in a typical inverted-T scar.

To perform the circumvertical mastopexy combined with silicone implants, the operation commenced with an inframammary approach to prepare the pocket for the implant. This was subpectoral for 15 patients, and prepectoral in one. Afterwards, a sizer was inserted and the incision in the inframammary crease was temporarily closed. The patient's upper body was then elevated to determine the amount of excess skin, which was then marked in a typical anchorshaped pattern. In a supine position, the anchor-shaped pattern was deepithelialized and the final silicone implant inserted. The margins of the remaining skin envelope were brought together and sutured plane-by-plane which resulted 

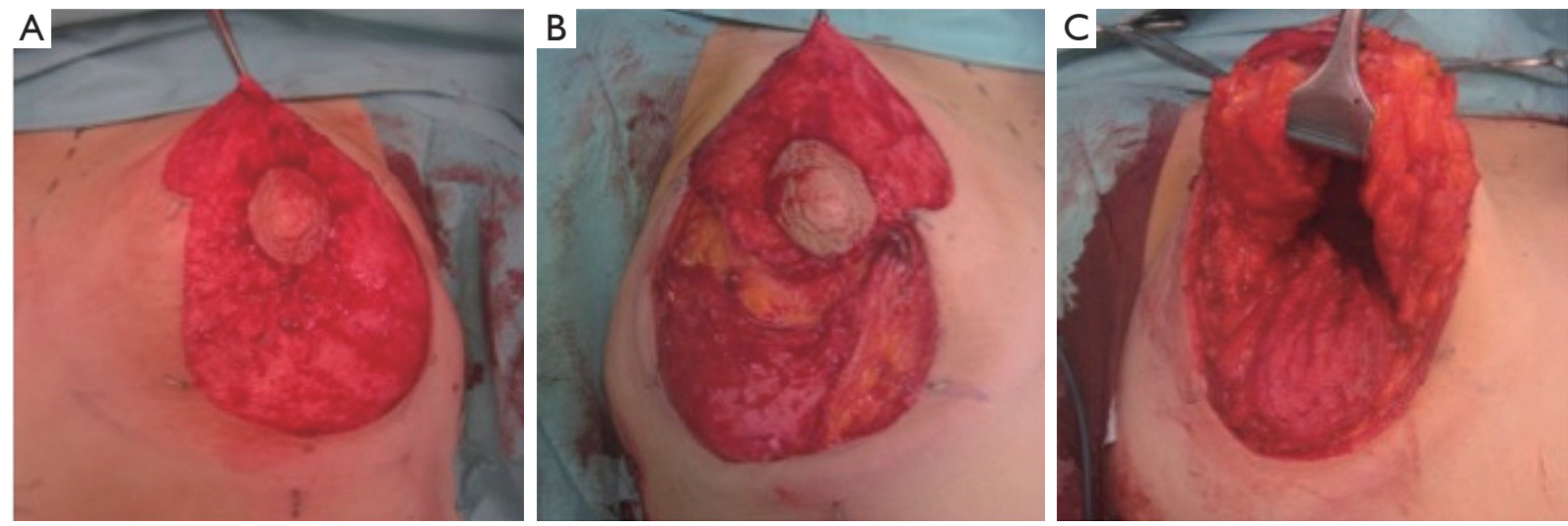

Figure 1 Surgical technique. (A) Deepithelialization of the Wise pattern; (B) horizontal incision of the dermis about one centimeter below the areola; (C) flap design before preparation of pectoralis muscle sling.

in either a vertical scar alone or an inverted-T scar.

\section{Photometric measurements}

Photometric measurements of lateral views were performed as described by Eric Swanson (11). Briefly, photographs were orientation-matched, and calibration was performed using an average upper arm length of $32.5 \mathrm{~cm}$ (12). Afterwards, a vertical line was dropped at the level of the suprasternal notch to mark the posterior breast margin. Then, a horizontal line was drawn at the level of maximum postoperative breast projection (Mpost). Area calculations above and below this plane allow for lower pole area (LPA) calculation as well as upper pole area (UPA) before and after surgery. To assess the level of upper pole projection (UPP) the distance between the level of breast projection and the sternal notch was bisected. The level of the lower pole (LP) was defined by the distance between the lowest point of the breast and maximum postoperative breast projection. Additional calculations allow for breast mound elevation (BME) determination, which was the difference between the level of maximum preoperative breast projection (Mpre) and Mpost.

Additionally, an assessment of the upper pole contour was performed in all patients. To do so, a diagonal line was drawn at the level of UPP, and the contour of the upper pole was compared with the line and divided into the following categories: linear, concave and convex.

\section{BREAST-Q}

Application of the BREAST-Q score was in accordance with the agreement with MAPI Trust (http://www.mapitrust.org/). During follow-ups, the BREAST-Q score was obtained with the German version of the BREAST-Q postaugmentation module. Questionnaires were completed anonymously by all patients. For our study, the postaugmentation module ideally fit the patients that wished to have a more youthful and voluminous breast. Due to a history of breast cancer, two patients were excluded from the BREAST-Q survey.

The questionnaire was separated in two parts and consisted of 88 questions in total: (I) patient satisfaction and (II) health-related quality of life. The satisfaction domain included satisfaction with breasts, outcome, information, surgeon, medical team, and office team. Quality of life domain included psychosocial, sexual, and physical wellbeing. Twelve questions specifically referring to silicone implants were excluded to allow for a valid comparison between both groups. The remaining majority of 76 responses were ranged on a Likert-like scale. Patients' responses to each scale items were transformed using the Q-Score scoring software.

\section{Statistics}

Statistical analysis was performed using descriptive and summary statistics to identify a central tendency. Data was analyzed using Microsoft ${ }^{\circledR}$ Excel Version 14.3.6. (Microsoft Corp., Redmond, WA, USA) and GraphPad Prism Version 7.04 (GraphPad, La Jolla, CA, USA).

The BREAST-Q score was calculated using the Q Score Excel template and corresponding Q Score program that converts raw survey scores from 1 to 3 or 5 in 
Table 1 Patient demographics

\begin{tabular}{lccc}
\hline Demographics & Augmentation-mastopexy $(\mathrm{N}=16)$ & Mastopexy $(\mathrm{N}=18)$ & $\mathrm{P}$ value \\
\hline Age, y (mean $\pm \mathrm{SD})$ & $39 \pm 11$ & $36 \pm 11$ & 0.8 \\
$\mathrm{BMI}, \mathrm{kg} / \mathrm{m}^{2}$ (mean $\left.\pm \mathrm{SD}\right)$ & $24 \pm 3$ & $25 \pm 3$ & 0.5 \\
Smoker, N [\%] & $5[31]$ & $8[44]$ & 0.4 \\
Previous operations, N [\%] & $5[31]$ & $6[33]$ & 0.9 \\
Follow-up, y, mean (range) & 4 (range, 1-6.6) & 3.8 (range, 3.3-7.3) & 0.4 \\
\hline
\end{tabular}
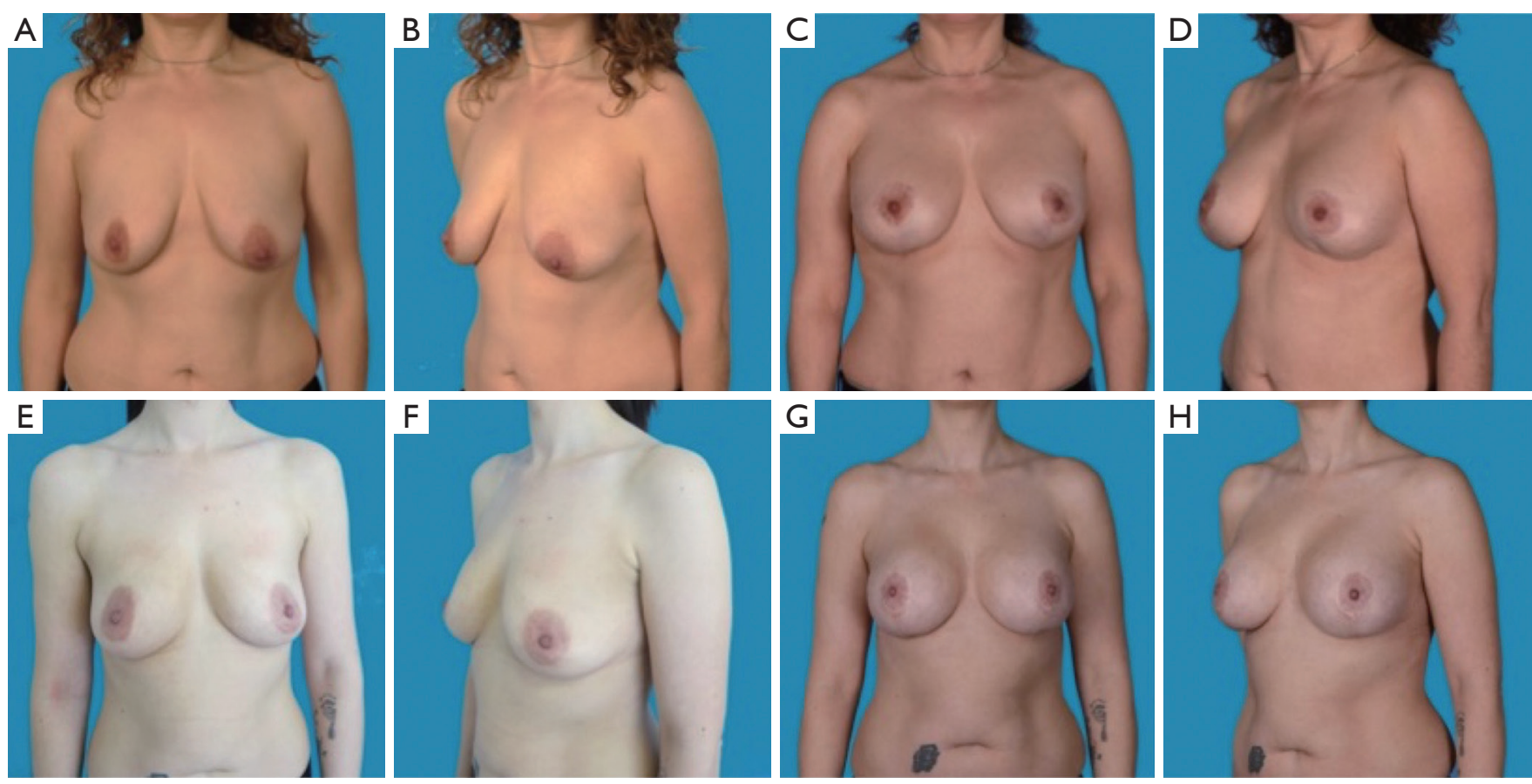

Figure 2 Two representative patients with breast ptosis (A,B: $35 \mathrm{yrs}$; E,F: 34 yrs) and involution of the breast after pregnancy before and 3 years after receiving a mastopexy with silicone implants $(\mathrm{C}, \mathrm{D}, \mathrm{G}, \mathrm{H})$ are shown.

continuous scores, thus generating a total score ranging from 0 to 100 . In order to analyze whether data shows a normal distribution, we performed D'Agostino-Pearson and Shapiro Wilk test. Given a $\mathrm{P}$ value $>0.05$, normal distribution was assumed for all further statistical tests. An unpaired $t$-test was performed to analyze the significance of changes in mean scores between both patient groups. The group test analysis was achieved by a Chi-square test. A P value of $<0.05$ was defined significant.

\section{Results}

The study was conducted with 34 patients (Table 1). Of these, 16 patients received an augmentation-mastopexy (Figure 2) and 18, a mastopexy (Figure 3). The average follow-up time was 4 years (range, 1-7.3 years). The mean age was $39 \pm 11$ in the augmentation-mastopexy versus $36 \pm 11$ years in the mastopexy group.

The most prevalent reason to have surgery was breast ptosis, which was preoperatively graded using the grading scale described by Regnault (Table 2) (13). In two patients, indication for surgery was due to implant-ruptures, which were diagnosed by preoperative magnetic resonance imaging. Seven additional patients presented with capsular contracture after esthetic breast augmentation. Of those, three patients received an implant removal followed by 

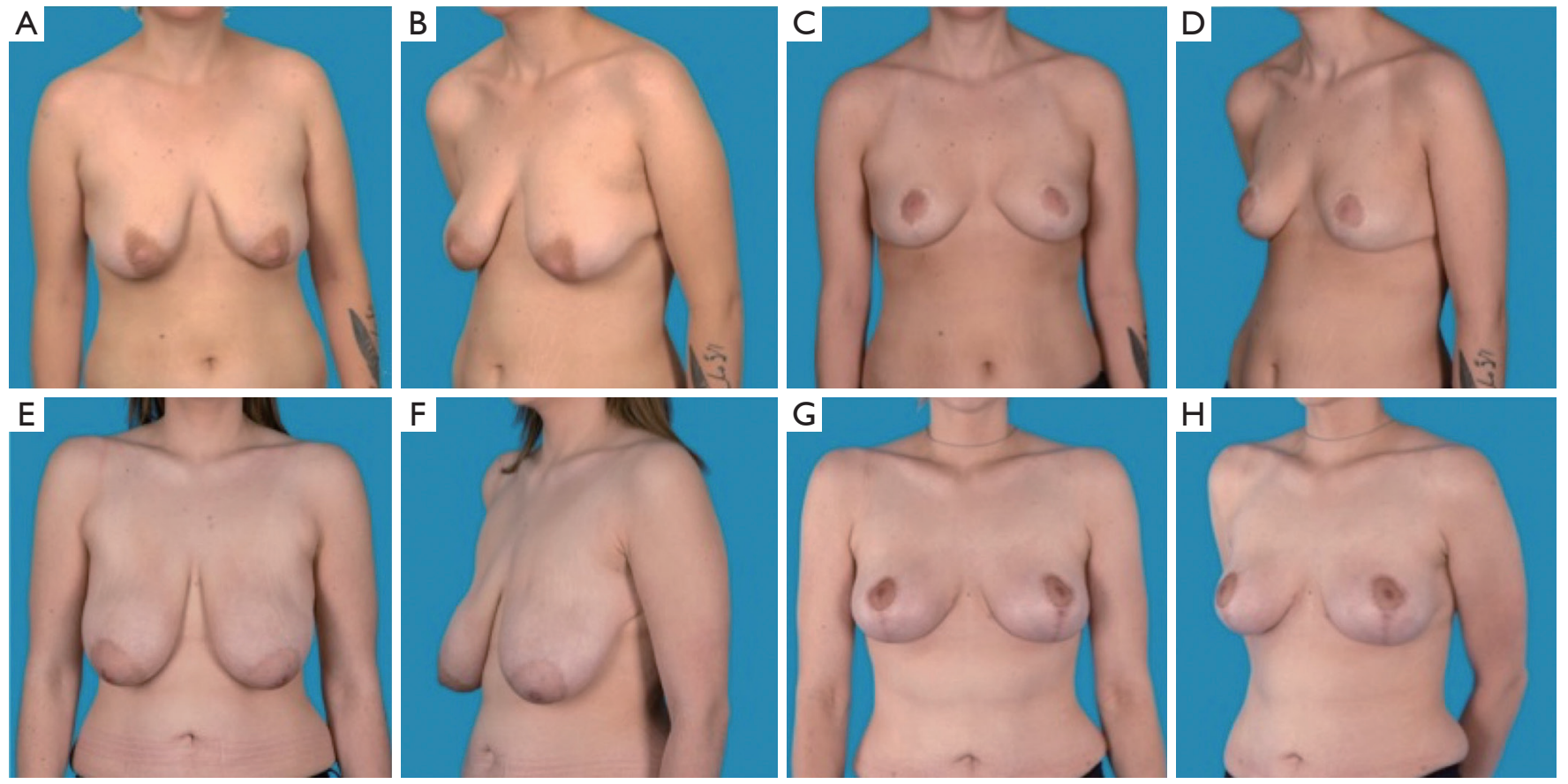

Figure 3 Two representative patients with breast ptosis before (A,B: $29 \mathrm{yrs}$; E,F: $25 \mathrm{yrs}$ ) and after receiving a mastopexy with an autoimplant (C,D: 27 months postoperative; G,H: 9 months postoperative) are shown.

Table 2 Diagnosis

\begin{tabular}{lcc}
\hline Diagnosis & Augmentation-mastopexy $(\mathrm{N}=16), \mathrm{N}[\%]$ & Mastopexy (N=18), N [\%] \\
\hline Mild breast ptosis & $4[25]$ & $3[17]$ \\
Moderate breast ptosis & $4[25]$ & $6[33]$ \\
Severe breast ptosis & $2[13]$ & $4[22]$ \\
Implant-associated complications & $6[36]$ & $3[17]$ \\
Breast carcinoma & $0[0]$ & $2[11]$ \\
\hline
\end{tabular}

mastopexy (17\%), whereas in the remaining four patients implant exchange and mastopexy were performed. Two patients $(11 \%)$ requested a mastopexy due to breast asymmetry after breast reconstruction.

The most common complication in our patient collective was a delayed wound healing of $>30$ days that occurred in four patients (22\%) after a mastopexy and in three patients (19\%) after an augmentation-mastopexy (Table 3). They were all located at the T-junction and classified as minor wound dehiscence due to an affected area below $0.5 \mathrm{~cm}$. They all healed by secondary intention without further complications. In both groups, one patient presented a postoperative hematoma that required reoperation only after the augmentation-mastopexy.
The majority of patients reported an unaffected sensitivity of the nipple areola complex regardless of the type of procedure. In the mastopexy group, five patients (28\%) developed widened scars and three patients (17\%) showed a slight breast volume asymmetry that was assessed during standardized breast measurements. After augmentation-mastopexy, widened scars (13\%) as well as breast volume asymmetry (13\%) were seen in two patients. A recurrent slight ptosis was observed in two patients (11\%) at year 6 after mastopexy, and in one patient $(6 \%)$ within 4 years after augmentation-mastopexy. Three years after having received an augmentation-mastopexy, two patients (13\%) developed a bottoming out.

The preoperatively assessed SNN was decreased in most 
Table 3 Complications

\begin{tabular}{|c|c|c|c|}
\hline Complications & Augmentation-mastopexy (N=16), N [\%] & Mastopexy (N=18), N [\%] & $P$ value \\
\hline Hematoma & $1[6]$ & $1[6]$ & 0.9 \\
\hline Seroma & $0[0]$ & $1[6]$ & 0.3 \\
\hline Infection & $0[0]$ & $0[0]$ & - \\
\hline
\end{tabular}

${ }^{\dagger}$, not healed within 30 days.

of the patients. The mean difference of $\mathrm{SNN}$ was $4.2 \pm 0.55$ $\mathrm{cm}$ after mastopexy and $1.5 \pm 0.23 \mathrm{~cm}$ after augmentationmastopexy, revealing a statistical significance $(\mathrm{P}=0.0001)$.

Based on photometric measurements (Figure 4), BME was analyzed in both groups in terms of a linear, concave or convex shape of breasts upper pole (Table 4, Figure 5). Statistical analysis revealed a significant difference of the breast's upper pole between both groups $(\mathrm{P}=0.01)$. Augmentation-mastopexy resulted in higher dimensions of the upper pole as well as maximum breast projection. The LP level showed a greater elevation after mastopexy. Interestingly, the mean of preoperative upper pole and maximum breast projection was almost similar in both groups.

The BREAST-Q post-augmentation module (Table 5) showed statistical significance in the following three categories: breast satisfaction $(\mathrm{P}=0.03)$, satisfaction with the outcome $(\mathrm{P}=0.009)$ and satisfaction with the surgeon $(\mathrm{P}=0.02)$. Given the different reasons to have surgery, an additional group-matched analysis was performed. While a tendency towards a higher satisfaction after mastopexy was observed, the difference was not statistically different for patients with breast ptosis. However, patients that had implant removal followed by mastopexy showed significantly higher satisfaction scores $(\mathrm{P}=0.007)$.

The mean scores of psychosocial well-being and physical well-being revealed a difference of nine points in favor for mastopexy that were not statistically significant $(\mathrm{P}=0.08$; $\mathrm{P}=0.15$ ). The mean scores of the remaining categories including sexual well-being, satisfaction with information, satisfaction with medical team, and satisfaction with the office staff were comparable.

\section{Discussion}

The applied BREAST-Q post-augmentation module allowed for a reliable comparison of patient satisfaction after augmentation-mastopexy and mastopexy, especially in regards to a more firm, youthful looking, and voluminous breast. Furthermore, the mean follow-up of 4 years allowed us to assess long-term satisfaction, which is crucial in validating a surgical breast procedure. Although our study is based on a small sample size, the comparison between both techniques regarding patient satisfaction has not been analyzed or reported yet elsewhere.

Traditionally, mastopexy was performed using primarily skin excision techniques such as crescent, periareolar, circumvertical, and inverted T-designs. However, since many patients seek restoration of the upper pole fullness, surgical techniques with an additional repositioning of an inferiorly, superiorly or central based glandular pedicle were introduced. Since the first reports of autologous parenchymal flaps in the 1970s $(14,15)$, numerous techniques with only subtle variations were published. These include variations regarding incision patterns, orientation of the pedicle and fixation maneuver (2-4,15-17). Further techniques propose the additional use of tissue from the back, referred to as thoracic wall flaps, that are especially popular in massive weight loss patients $(5,18)$. Given these numerous surgical techniques, the superior technique regarding long-term stability remains a matter of debate. In 2011, Swanson reviewed 82 publications on mastopexy based on his proposed measuring system to quantitate and compare results. He claimed that any methods to increase upper pole fullness or projection, such as fascial sutures and glandular repositioning, generally did not maintain shape in the long term (19). More recent studies include cohorts with $27-184$ patients and analyze the outcome after mastopexy with an inferiorly-based parenchymal flap within one year after surgery. Similar to our results, a mean nipple elevation between 4 to $8 \mathrm{~cm}$ has been reported. A natural sagging of the breast of $1 \mathrm{~cm}$ within the first year of surgery 

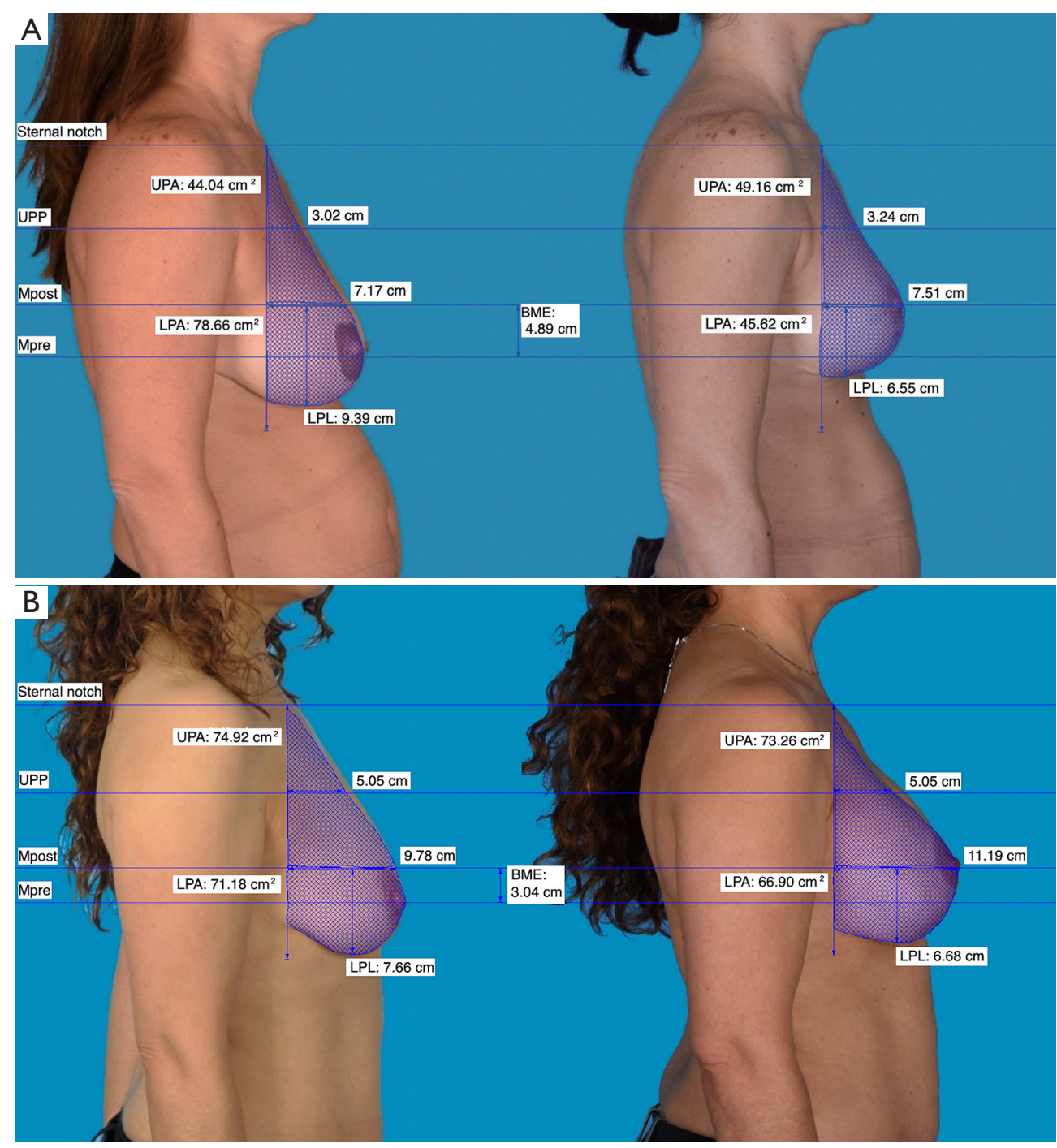

Figure 4 Orientation-matched lateral views of patients before and after mastopexy either with autoimplants (A: 38 yrs, 1 year postoperative) or silicone implants (B: $35 \mathrm{yrs}, 3$ years postoperative) are shown. Breast mound elevation (BME) goes along with an increased maximum breast projection [maximum preoperative breast projection (Mpre)]; maximum postoperative breast projection (Mpost) in both patients after surgery. Upper pole projection (UPP) is slightly increased in patient A. LPA, lower pole area; UPA, upper pole area; LPL, lower pole level.

was observed. Nevertheless, neither upper pole fullness, patient satisfaction nor long-term results after more than 1 year was assessed by any of the studies $(1,3,4,9)$.

Augmentation mastopexy was first described by Gonzalez-Ulloa in 1960. Since then, several algorithms have emerged to assist the surgeon in deciding whether to stage the operation as two separate individual operations or to perform them as single procedure. Further considerations include implant size, implant placement, implant shape, skin incision pattern and the use of a mesh (20). We performed a single stage augmentation-mastopexy in all patients and based our decision on the breasts vertical excess as proposed by Lee et al. (21). Similar to others, we observed no increased incidence of postoperative complications compared with the mastopexy group $(22,23)$.

The desired tightening of the skin typically encourages delayed wound healing which represents the most common complication in both mastopexy alone as well as simultaneous augmentation-mastopexy (24). These occurred at the convergence of the flaps in the inverted-T skin scar, 
Table 4 Photometric measurements

\begin{tabular}{|c|c|c|c|}
\hline Parameter & Augmentation-mastopexy $(\mathrm{N}=10)$ & Mastopexy $(\mathrm{N}=8)$ & $P$ value \\
\hline Postoperative UPP cm (range) & $6.2(4.8-7.6)$ & $5.3(3.2-7.7)$ & 0.5 \\
\hline Mpre cm (range) & $9.3(6.1-12.5)$ & $9.2(7.2-12.9)$ & \\
\hline Mpost cm (range) & $11.1(9.6-13.3)$ & $9.5(7.5-13.3)$ & 0.2 \\
\hline Difference in LPL (mean \pm SD) & $1.2 \pm 1.8$ & $2.9 \pm 1.4$ & 0.6 \\
\hline Linear UPF (\%) (N=34) & 38 & 55 & \\
\hline Concave UPF (\%) (N=34) & 13 & 39 & $0.01^{*}$ \\
\hline Convex UPF (\%) (N=34) & 50 & 6 & \\
\hline
\end{tabular}

*, statistical significance defined as $\mathrm{P}<0.05$; UPP, upper pole projection; Mpre, maximum preoperative breast projection; Mpost, maximum postoperative breast projection; BME, breast mound elevation; LPL, lower pole level; UPF, upper pole fullness.
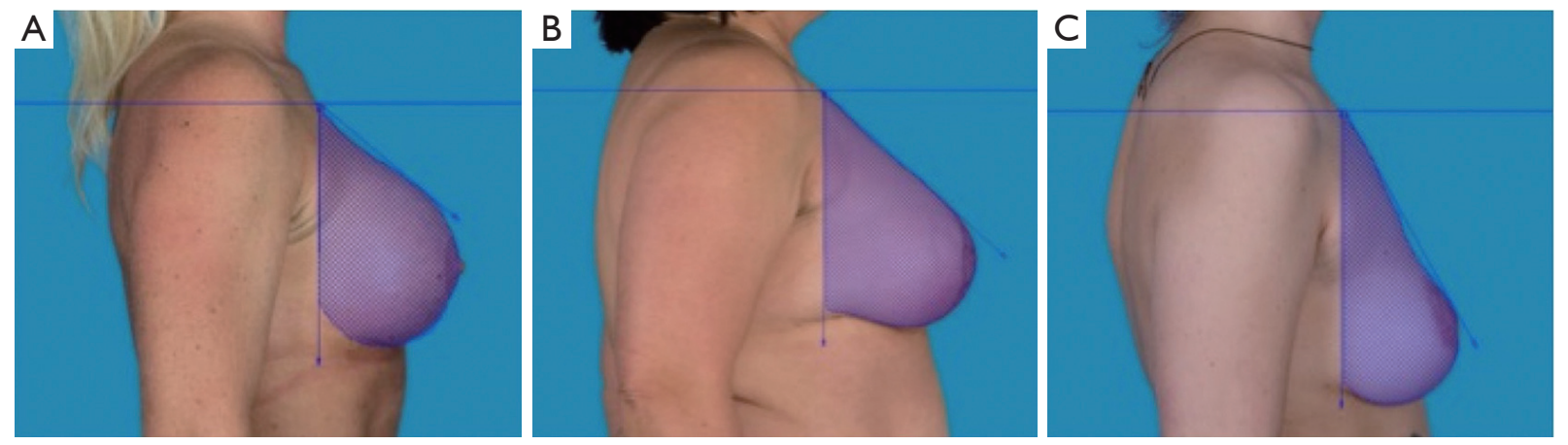

Figure 5 The upper pole fullness was assessed by the contour of the upper pole of the breast between the upper chest takeoff and the maximum point of breast projection. It was either convex (A: 33 yrs, 5 years postoperative), linear (B: 52 yrs, 2 years postoperative) or concave (C: 25 yrs, 2 years postoperative).

where the tension on the skin flaps is usually the highest. Compared with other studies, the incidence of delayed wound healing was quite high $(1,3,24)$. This however, might be explained by a high number of patients that had previous breast operations as well as the fact that almost half of the patients were smokers.

BREAST-Q score of patients that had undergone mastopexy revealed a remarkable difference in satisfaction with breast and outcome, therefore implying a higher overall long-term satisfaction, although long-term results and complication rates were comparable. Notably, groupmatched analysis showed significantly higher satisfaction scores after implant removal followed by mastopexy.

To our knowledge, literature regarding patient satisfaction after the use of an autoimplant is limited to a few studies. Similar to our findings, Gurunluoglu et al. revealed an improvement of satisfaction with breast $(75.2 \pm 11.0)$, psycho-social $(66.3 \pm 10.2)$, and sexual well-being $(65.7 \pm 16.3)$ after breast implant removal and "autoaugmentation" using an inferior pedicle dermoglandular flap including 20 patients (25). A further study observed statistically significant improvements in satisfaction with breast (89.2 \pm 20.7$)$, psychosocial $(86.1 \pm 22.7)$, sexual $(73.3 \pm 16.6)$ and physical well-being $(86.3 \pm 14.6)$ after any breast reshaping procedures including "autoaugmentation", breast reduction, mastopexy and silicone implants, however satisfaction scores were not distinguished between the different reshaping techniques (26).

Recently, Mundy et al. published normative data for the BREAST-Q augmentation module that allows further 
Table 5 BREAST-Q

\begin{tabular}{lccc}
\hline Domains & $\begin{array}{c}\text { Augmentation-mastopexy (N=16), } \\
\text { (mean } \pm \text { SD) }\end{array}$ & $\begin{array}{c}\text { Mastopexy (N=16), } \\
(\text { mean } \pm \text { SD) }\end{array}$ & P value \\
\hline Satisfaction with breast & $55 \pm 16$ & $69 \pm 18$ & $0.03^{*}$ \\
Ptosis grade I & $72 \pm 19$ & $78 \pm 22$ & 0.73 \\
Ptosis grade II & $48 \pm 7$ & $60 \pm 10$ & 0.10 \\
Ptosis grade III & $58 \pm 16$ & $73 \pm 22$ & 0.50 \\
Implant-associated complications & $47 \pm 7$ & $72 \pm 11$ & $0.007^{*}$ \\
Satisfaction with outcome & $48 \pm 26$ & $71 \pm 18$ & $0.009^{*}$ \\
Ptosis grade I & $69 \pm 26$ & $76 \pm 9$ & 0.70 \\
Ptosis grade II & $40 \pm 14$ & $64 \pm 18$ & 0.08 \\
Ptosis grade III & $74 \pm 26$ & $79 \pm 24$ & 0.85 \\
Implant-associated complications & $30 \pm 15$ & $67 \pm 4$ & $0.007^{*}$ \\
Psychosocial well-being & $55 \pm 28$ & $64 \pm 22$ & 0.08 \\
Sexual well-being & $56 \pm 31$ & $58 \pm 27$ & 0.80 \\
Physical well-being & $81 \pm 15$ & $90 \pm 16$ & 0.15 \\
Satisfaction with information & $63 \pm 21$ & $65 \pm 16$ & 0.75 \\
Satisfaction with surgeon & $70 \pm 18$ & $88 \pm 18$ & $0.02^{*}$ \\
Satisfaction with medical team & $82 \pm 21$ & $88 \pm 24$ & 0.50 \\
Satisfaction with office staff & $88 \pm 20$ & $89 \pm 22$ & 1.00 \\
\hline
\end{tabular}

${ }^{*}$, statistical significance defined as $\mathrm{P}<0.05$.

comparison with women not actively seeking breast augmentation (19). Given the mean scores for breast satisfaction $(54 \pm 19)$, sexual well-being $(49 \pm 20)$, and physical well-being $(86 \pm 15)$. Although, the small sample size of our study limits statistical power, we could observe similar scores in patients after augmentation-mastopexy (27). In fact, patients that had undergone a mastopexy revealed significantly greater scores, therefore indicating a higher long-term satisfaction. A similar phenomenon has been reported for patients receiving breast reconstruction; although long term patient satisfaction was significantly higher after breast reconstruction with autologous tissues compared to breast reconstruction with silicone implants (28-31). While breast implants cause a foreign body reaction that can lead to capsular contracture and results in poor aesthetic results, and pain in the long run, autologous tissue offers more stable and lasting results. Incidence rates of capsular contracture increase with the follow-up period and range from $5.9 \%$ to $8.1 \%$ in studies with an average follow -up of 3 years. Interestingly, after an average of 19 years,
$62 \%$ of patients are diagnosed with a clinically significant capsular contracture that requires implant exchange and effortful capsulectomy $(32,33)$. We hypothesize that the knowledge of the high risk to undergo further operations for implant exchange might lead to a remarkable decrease of patients' satisfaction. Moreover, patients that had breast implants in the past will experience the capability of autologous tissue to create a soft and natural looking breast which might serve as an explanation for the significantly higher satisfaction scores.

On the other hand, the higher prevalence of moderate to severe ptosis in the mastopexy group needs to be considered. Thus, expectations might have differed remarkably from those having a rather mild to moderate ptosis resulting in dissatisfaction and lower scores. The significantly higher surgeon satisfaction that was observed in the mastopexy group, also hints towards an overall more content patient collective.

Photometric measurements revealed a rather linear or concave shaped upper pole and a greater elevation of 
breast mound and LPL that was in line with a significantly higher difference of pre- and postoperative SNN after a mastopexy. This can be explained by a greater number of patients with moderate to severe breast ptosis and thus a higher preoperative SNN in the mastopexy group. A smaller overall breast volume, as well as less weight of the autoimplant which is formed and moved upwards, compared with the additional weight and stress of silicone implants, might serve as a further explanation.

Augmentation-mastopexy resulted in a rather convex contour of the upper pole. In contrast to other studies, where the convex contour was described as the ideal breast shape, satisfaction with breasts were significantly lower in our patient collective $(11,12,34)$. In the past, great differences in breast shape preferences, especially with regard to the area of culture have been reported. In 2003, Hsia et al. conducted a study in the United States including plastic surgeons, patients seeking breast augmentation, and lay people. Whereas plastic surgeons and lay people rated concave upper pole contours significantly higher and to be more natural, the patients cohort gave higher scores for convex contours (35). A further study found significant differences in ideal upper pole fullness among surgeons across different countries with a preference for moderate upper pole fullness in the United States and a preference for a high degree of upper pole fullness among German surgeons. The authors therefore conclude that an ideal shape of the upper pole does not exist and has to be evaluated in each patient before breast surgery (36). These findings are confirmed by our study.

\section{Conclusions}

In conclusion, the combined technique of a mastopexy with an autoimplant is a superior alternative for patients seeking more youthful looking breasts with excellent longterm results and a higher overall long-term satisfaction. Nonetheless, proper patient selection is critical in preventing unrealistic expectations. It is important to take note of the breasts' volume, previous operations, and the patients' preferences in effort to meet their desired outcome. In this context, it must be emphasized that an augmentation of a breast with a higher degree of upper pole fullness can only be achieved by silicone implants. However, the main advantage of an autoimplant is the use of autologous tissue to improve breast ptosis and overall projection.

\section{Acknowledgments}

We would like to thank Dr. Michelle McLuckie, PhD for her assistance during the preparation process and review and revisions of the final manuscript. This study received no financial support.

\section{Footnote}

Conflicts of Interest: The authors have no conflicts of interest to declare.

Ethical Statement: The authors are accountable for all aspects of this work in ensuring that questions related to the accuracy or integrity of any part of the work are appropriately investigated and resolved. The authors have no conflict of interest to declare. This study was approved by the Cantonal Ethics Committee of Zurich, Switzerland (Ethical approval No. 2017-01333). All patients gave written consent prior to study participation.

\section{References}

1. Kirwan L, Wazir U, Mokbel K. Breast AutoAugmentation: A Versatile Method of Breast Rehabilitation-A Retrospective Series of 107 Procedures. Arch Plast Surg 2015;42:438-45.

2. Graf R, Biggs TM. In search of better shape in mastopexy and reduction mammoplasty. Plast Reconstr Surg 2002;110:309-17; discussion 318-22.

3. Nava M, Rancati A, Rocco N, et al. Improving aesthetic outcomes in mastopexy with the "autoprosthesis" technique. Gland Surg 2017;6:141-7.

4. Ors S. Autoaugmentation Mastopexy Modification Prevents Bottoming-Out Deformity and Areola Distortion: A Preliminary Report. Aesthetic Plast Surg 2016;40:497-506.

5. Rubin JP, Khachi G. Mastopexy after massive weight loss: dermal suspension and selective auto-augmentation. Clin Plast Surg 2008;35:123-9.

6. Hillard C, Fowler JD, Barta R, et al. Silicone breast implant rupture: a review. Gland Surg 2017;6:163-8.

7. Rocco N, Rispoli C, Moja L, et al. Different types of implants for reconstructive breast surgery. Cochrane Database Syst Rev 2016;(5):CD010895.

8. Kirwan L. Breast autoaugmentation. Can J Plast Surg 2007;15:73-6.

9. Honig JF, Frey HP, Hasse FM, et al. Autoaugmentation 
mastopexy with an inferior-based pedicle. Aesthetic Plast Surg 2009;33:302-7.

10. Pusic AL, Reavey PL, Klassen AF, et al. Measuring patient outcomes in breast augmentation: introducing the BREAST-Q Augmentation module. Clin Plast Surg 2009;36:23-32, v.

11. Swanson E. A measurement system for evaluation of shape changes and proportions after cosmetic breast surgery. Plast Reconstr Surg 2012;129:982-92.

12. Westreich M. Anthropomorphic breast measurement: protocol and results in 50 women with aesthetically perfect breasts and clinical application. Plast Reconstr Surg 1997;100:468-79.

13. Regnault P. Breast ptosis. Definition and treatment. Clin Plast Surg 1976;3:193-203.

14. Ribeiro L. A new technique for reduction mammaplasty. Plast Reconstr Surg 1975;55:330-4.

15. McKissock PK. Reduction mammaplasty with a vertical dermal flap. Plast Reconstr Surg 1972;49:245-52.

16. Kim P, Kim KK, Casas LA. Superior pedicle autoaugmentation mastopexy: a review of 34 consecutive patients. Aesthet Surg J 2010;30:201-10.

17. Hester TR Jr, Bostwick J 3rd, Miller L, et al. Breast reduction utilizing the maximally vascularized central breast pedicle. Plast Reconstr Surg 1985;76:890-900.

18. Graf R, Reis de Araujo LR, Rippel R, et al. Reduction mammaplasty and mastopexy using the vertical scar and thoracic wall flap technique. Aesthetic Plast Surg 2003;27:6-12.

19. Swanson E. A retrospective photometric study of 82 published reports of mastopexy and breast reduction. Plast Reconstr Surg 2011;128:1282-301.

20. Sarosiek K, Maxwell GP, Unger JG. Getting the Most Out of Augmentation-Mastopexy. Plast Reconstr Surg 2018;142:742e-59e.

21. Lee MR, Unger JG, Adams WP Jr. The tissue-based triad: a process approach to augmentation mastopexy. Plast Reconstr Surg 2014;134:215-25.

22. Swanson E. Prospective comparative clinical evaluation of 784 consecutive cases of breast augmentation and vertical mammaplasty, performed individually and in combination. Plast Reconstr Surg 2013;132:30e-45e.

23. Khavanin N, Jordan SW, Rambachan A, et al. A systematic review of single-stage augmentation-mastopexy. Plast Reconstr Surg 2014;134:922-31.

24. Weissman O, Winkler E, Teot L, et al. Treatment of wounds following breast reduction and mastopexy with subsequent wound dehiscence with charged polystyrene microspheres. Wounds 2014;26:37-42.
25. Gurunluoglu R, Sacak B, Arton J. Outcomes analysis of patients undergoing autoaugmentation after breast implant removal. Plast Reconstr Surg 2013;132:304-15.

26. Vindigni V, Scarpa C, Tommasini A, et al. Breast Reshaping Following Bariatric Surgery. Obes Surg 2015;25:1735-40.

27. Mundy LR, Homa K, Klassen AF, et al. Normative Data for Interpreting the BREAST-Q: Augmentation. Plast Reconstr Surg 2017;139:846-53.

28. Durry A, Baratte A, Mathelin C, et al. Patients' satisfaction after immediate breast reconstruction: Comparison between five surgical techniques. Ann Chir Plast Esthet 2019;64:217-23.

29. Fracon S, Renzi N, Manara M, et al. Patient Satisfaction after Breast Reconstruction: Implants Vs. Autologous Tissues. Acta Chir Plast 2018;59:120-8.

30. Pirro O, Mestak O, Vindigni V, et al. Comparison of Patient-reported Outcomes after Implant Versus Autologous Tissue Breast Reconstruction Using the BREAST-Q. Plast Reconstr Surg Glob Open 2017;5:e1217.

31. Pusic AL, Matros E, Fine N, et al. Patient-Reported Outcomes 1 Year After Immediate Breast Reconstruction: Results of the Mastectomy Reconstruction Outcomes Consortium Study. J Clin Oncol 2017;35:2499-506.

32. Marques M, Brown SA, Oliveira I, et al. Long-term followup of breast capsule contracture rates in cosmetic and reconstructive cases. Plast Reconstr Surg 2010;126:769-78.

33. Holmich LR, Breiting VB, Fryzek JP, et al. Long-term cosmetic outcome after breast implantation. Ann Plast Surg 2007;59:597-604.

34. Swanson E. Ideal breast shape: women prefer convexity and upper pole fullness. Plast Reconstr Surg 2015;135:641e-3e.

35. Hsia HC, Thomson JG. Differences in breast shape preferences between plastic surgeons and patients seeking breast augmentation. Plast Reconstr Surg 2003;112:31220; discussion 321-2.

36. Broer PN, Juran S, Walker ME, et al. Aesthetic breast shape preferences among plastic surgeons. Ann Plast Surg 2015;74:639-44.

Cite this article as: Grünherz L, Burger A, Giovanoli P, Lindenblatt N. Long-term results measured by BREAST-Q reveal higher patient satisfaction after "autoimplant-mastopexy" than augmentation-mastopexy. Gland Surg 2019;8(5):516-526. doi: 10.21037 /gs.2019.09.05 